\title{
FREE BOUNDARY PROBLEMS IN CONTROLLED RELEASE PHARMACEUTICALS. I: DIFFUSION IN GLASSY POLYMERS*
}

\author{
DONALD S. COHEN† AND THOMAS ERNEUX $\ddagger$
}

\begin{abstract}
This paper formulates and studies two different problems occurring in the formation and use of pharmaceuticals via controlled release methods. These problems involve a glassy polymer and a penetrant, and the central problem is to predict and control the diffusive behavior of the penetrant through the polymer. The mathematical theory yields free boundary problems which are studied in various asymptotic regimes.
\end{abstract}

Key words. free boundaries, perturbations, polymers, controlled release

AMS(MOS) subject classifications. 35B25, 35F99, 35K20, 85A51

1. Introduction. A central problem of controlled release technology in the pharmaceutical industry is to combine an active agent (the drug or medicine) with its carrier (a polymer) in an economical manner to achieve a release profile that best fits the situation at hand. The resulting devices may be swallowed, smelled, surgically implanted, rubbed on, taped on, or strapped on to selectively reach virtually every part of the body. Many problems or disadvantages associated with conventional usage of pharmaceuticals can be eliminated or greatly alleviated with these new methods: these include systemic effects, patient compliance, and undesirable, inaccurate, fluctuating dosages.

The physical process common to most of controlled release technology is diffusion [9]. The drugs are released by diffusion alone or by diffusion in combination with other mechanisms. The fabrication and storage of the pharmaceuticals also involves diffusive processes. Many new synthetic polymers are used as separating membranes, encapsulating devices, or impregnated matrices, and many of these have intriguing and fascinating diffusive properties quite unlike anything seen before. Clearly, we need to understand how drugs move through membranes, settle out of ointments and creams, or move through a pill, tablet, or patch. That is, we must understand how to accurately and precisely control rates, duration, and sites of release. Of particular interest is the time history of a diffusing front of a penetrant through the polymer. Flux rates are critical in the formation and storage of the device, and they are the determining factor in the prescription and administration of the pharmaceuticals.

According to classical diffusion theory, the position $x=s(t)$ of a moving boundary is given by $x=$ (constant) $t^{1 / 2}$. This follows from solving a so-called one-phase Stefan problem for the concentration $C(x, t)$ behind a moving boundary at position $x=s(t)$. We must find both $C(x, t)$ and $s(t)$, where $C$ satisfies the classical Fick's diffusion equation, $C_{t}=\left(D C_{x}\right)_{x}$, subject to various initial and boundary conditions involving $s(t)$. Here $D$ is the diffusivity of the medium.

* Received by the editors September 10, 1986; accepted for publication (in revised form) September $16,1987$.

$\dagger$ Department of Applied Mathematics, California Institute of Technology, Pasadena, California 91125. This work was supported in part by U.S. Army Research Office (Durham) under contract DAAG29-85-K-0092, by the National Science Foundation under grant DMS-8706642, and by the Department of Energy under grant 7405/ENG-36 at the Center for Nonlinear Studies at Los Alamos.

$\ddagger$ Department of Engineering Sciences and Applied Mathematics, Northwestern University, Evanston, Illinois 60208. This work was supported in part by the Air Force Office of Scientific Research under grant AFOSR85-0150 and the National Science Foundation under grant DMS-8701302. 
Since the work of Alfrey, Gurnee, and Lloyd [1], it has been generally recognized that in polymer-penetrant systems the motion of the penetrant front can proceed according to laws considerably different from the classical simple $t^{1 / 2}$ of Fickian diffusion, and many of these polymer-penetrant systems are of major importance in controlled release technology in the pharmaceutical industry. Furthermore, there is no simple alternative to the classical situation [2]-[5]. Although some global principles are starting to emerge, the fact is that there is no universal theory, and different models (theories, equations of motion) are needed for systems of one type which may differ considerably from those of other types of systems depending on type of polymer, type of diffusing substance, and ambient conditions.

In this paper, we consider two different problems involving polymer-penetrant systems of the type where the driving force is due to local kinetics at the moving interface. A large number of systems of this type together with experimental observations is listed in Astarita and Joshi [6]. The problems occur in the formation and storage of simple swelling controlled drug release systems without volume change [5], [7]. Our main result is that there is a transition from initial $t$ behavior (so-called Case II diffusion) to long time $t^{1 / 2}$ behavior (Fickian diffusion). For pharmaceutical applications it is desirable to maintain Case II behavior (also called zero-order release) as long as possible. Our asymptotic analysis shows the dependence on the various parameters of the problem. Our purpose is to find an optimal strategy for long time $t$ behavior.

In a companion paper, we then investigate a model for the swelling-controlled release of a drug and show how our previous study of polymer-penetrant interactions can be applied to obtain the history of release.

2. Constant reservoir of swelling solvent. A polymer film is exposed to a constant reservoir of a smaller molecule capable of diffusing in the film. As a result, a sharp interface appears in the polymer which separates a swollen rubbery region where the solvent concentration is high from a glassy region where the solvent concentration is almost zero. This swollen-glassy interface moves through the polymer with a velocity which is constant near time $T=0$, varies as $T^{-p}\left(0<p<\frac{1}{2}\right)$ at intermediate times and may or may not decay exponentially to zero as $T \rightarrow \infty$. Our main purpose is to describe mathematically this gradual change of the glass-gel transition front. To this end, we consider a class of diffusion problems for which the kinetics of the phase transition is taken into account. The models are based on the work by Astarita and Sarti [8] who have successfully analyzed a large number of polymer-penetrant systems. In this paper, we complete and extend their analysis by describing the complete history of the penetrant front.

We assume that either the diffusivity or the solubility (or both) of the solvent in the glassy phase of the polymer is zero and we take the diffusivity $D$ of the swollen polymer to be constant. The problem is then formulated as a one-phase free boundary problem for the concentration $C(X, T)$ of the penetrant and the position $S(T)$ of the interface:

$$
\begin{gathered}
C_{T}=D C_{X X}, \quad 0<X<S(T), \\
C=C_{0}>C^{*} \quad \text { at } X=0, \\
(C+K) S^{\prime}=-D C_{X} \quad \text { at } X=S(T), \\
S^{\prime}=k_{1}\left(C-C^{*}\right)^{n} \quad \text { at } X=S(T), \\
S(0)=0 .
\end{gathered}
$$


The subscripts $T$ and $X$ indicate partial derivatives and $S^{\prime}=d S / d t . k_{1}, K, D, C_{0}$, and $C^{*}$ are given constant parameters. Equation (2.1) is Fick's Diffusion Law for a one-dimensional system subject to a fixed concentration at $X=0((2.2))$. $C_{0}$ is the solubility of the solvent in the swollen polymer. Equation (2.4) describes the swelling kinetics as an interface reaction: the excess $C(X, T)-C^{*}$ over the equilibrium value $C^{*}$ drives the penetrant front at some finite rate. In this equation $k_{1}$ and $n$ represent phenomenological quantities. Equation (2.3) is a mass balance at the moving front. It is derived in the following way: the flux from the swelling region across a moving boundary is given by $-D C_{X}-C S^{\prime}$ and is assumed to be proportional to the flux generated by the interface region. Thus we have

$$
-D C_{X}-C S^{\prime}=k_{2}\left(C-C^{*}\right)^{n} \quad \text { at } X=S(T),
$$

and using (2.4), we obtain (2.3) with $K=k_{2} / k_{1}$.

The problem (2.1)-(2.5) can be considerably simplified if we introduce the following dimensionless independent and dependent variables:

$$
\begin{aligned}
& u(x, t)=\frac{C(X, T)-C^{*}}{C_{0}-C^{*}}, \quad L(t)=\frac{S(T)}{\beta}, \\
& t=\frac{T}{\alpha}, \quad x=\frac{X}{\beta}
\end{aligned}
$$

where

$$
\alpha=\frac{D\left(C_{0}-C^{*}\right)^{1-2 n}}{\bar{C} k_{1}^{2}}, \quad \beta=\frac{D\left(C_{0}-C^{*}\right)^{1-n}}{\bar{C} k_{1}}
$$

and

$$
\bar{C}=C^{*}+K
$$

Inserting (2.7) into (2.1)-(2.5), we obtain

$$
\begin{gathered}
u_{t}=\varepsilon^{-1} u_{x x}, \quad 0<x<L(t), \\
u=1 \quad \text { at } x=0, \\
(1+\varepsilon u) L^{\prime}=-u_{x} \quad \text { at } x=L(t), \\
L^{\prime}=u^{n} \quad \text { at } x=L(t), \\
L(0)=0,
\end{gathered}
$$

where $L^{\prime}=d L / d t$ and $\varepsilon$ is a control parameter defined by

$$
\varepsilon=\frac{C_{0}-C^{*}}{\bar{C}}>0
$$

We now investigate the solution of these equations for short times, long times and in the limit $\varepsilon \rightarrow 0$. Since in each case $L(t)$, the position of the moving boundary, must be expanded in terms of a small parameter, it will be mathematically convenient to consider

$$
y=\frac{x}{L(t)}
$$


as our new independent space variable. Then, (2.8)-(2.12) can be rewritten as

$$
\begin{gathered}
\varepsilon L^{2} u_{t}=u_{y y}+\varepsilon y L L^{\prime} u_{y}, \quad 0<y<1, \\
u=1 \quad \text { at } y=0, \\
(1+\varepsilon u) L L^{\prime}=-u_{y} \quad \text { at } y=1, \\
L^{\prime}=u^{n} \text { at } y=1, \\
L(0)=0 .
\end{gathered}
$$

2.1. Short time behavior (small $L$ ). For small $L$, we seek a solution of (2.15)-(2.19) by expanding $u$ and $L^{\prime}$ in power series in $L$

$$
\begin{gathered}
u(y, L)=u_{0}(y)+L u_{1}(y)+L^{2} u_{2}(y)+\cdots, \\
L^{\prime}(L)=G_{0}+L G_{1}+\cdots .
\end{gathered}
$$

We shall determine the functions $u_{0}(y), u_{1}(y) \cdots$ and the numbers $G_{0}, G_{1}, \cdots$ sequentially. The asymptotic form of (2.21) is suggested by the physics of the situation. That is, we expect that $L \sim t$ initially (i.e., $L^{\prime} \sim G_{0}=$ constant). Mathematically, this follows from (2.16) and (2.18) which we expect to dominate initially, so that as long as $n$ is finite, we see that $L^{\prime} \sim 1$ initially. After substituting (2.20) and (2.21) into (2.15)-(2.18) and equating like powers of $L$, we obtain a sequence of simple problems for $u_{0}, u_{1} \cdots G_{0}, G_{1} \cdots$. Solving the two first problems leads to the following results:

$$
\begin{gathered}
u(y, L)=1-L(1+\varepsilon) y+O\left(L^{2}\right), \\
L^{\prime}=1-n(1+\varepsilon) L+O\left(L^{2}\right) .
\end{gathered}
$$

Using the initial condition (2.19), we integrate (2.23) to obtain

$$
L+\frac{n}{2}(1+\varepsilon) L^{2}+O\left(L^{3}\right)=t
$$

Thus, using the definition (2.14) and equation (2.24) for the front position, we have

$$
u(x, L)=1-(1+\varepsilon) x+O\left(L^{2}\right), \quad 0<x<L
$$

and

$$
L(t)=t-\frac{n}{2}(1+\varepsilon) t^{2}+O\left(t^{3}\right)
$$

We conclude that for small times the interface moves like $t$. We also note that the corresponding concentration $u$ at the front $x=L$ decreases linearly with $t$.

2.2. Long time behavior (Large $L$ ). To determine the long time solution, it is reasonable to assume that as $t \rightarrow \infty$ the contribution of the interface kinetics becomes negligible and that the behavior of the concentration in the swollen phase is mainly Fickian. In other words, we assume that $L(t) \sim t^{1 / 2}$ and $u(y, t) \sim u(y)$ as $t \rightarrow \infty$. Note from (2.18) that the first assumption implies that $u(1, t)=O\left(L(t)^{-1 / n}\right)$ as $t \rightarrow \infty$. This suggests that we seek a solution in the form

$$
\begin{gathered}
u(y, \tau)=u_{0}(y)+\tau u_{1}(y)+\cdots \\
\left(L^{2}\right)^{\prime}=E_{0}+\tau E_{1}+\cdots
\end{gathered}
$$

where $\tau$ is a small parameter defined by

$$
\tau=L^{-1 / n} \rightarrow 0 \text { as } L \rightarrow \infty .
$$


After inserting (2.27) and (2.28) into (2.15)-(2.18), we equate the coefficients of each power of $\tau$ to zero. This leads to the following problem for $u_{0}(y)$ and $E_{0}$ :

$$
\begin{gathered}
0=u_{0 y y}+\frac{\varepsilon y}{2} E_{0} u_{0 y}, \quad 0<y<1, \\
u_{0}=1 \text { at } y=0, \\
\left(1+\varepsilon u_{0}\right) \frac{1}{2} E_{0}=-u_{0 y} \quad \text { at } y=1, \\
u_{0}=0 \text { at } y=1 .
\end{gathered}
$$

Equation (2.30) with (2.31) and (2.33) is easily solved. We find that

$$
u_{0}(y)=1-\frac{\operatorname{erf}(y / \gamma)}{\operatorname{erf}(1 / \gamma)}
$$

where

$$
\gamma=2\left(\varepsilon E_{0}\right)^{-1 / 2}
$$

Then (2.32) with (2.33) implies that

$$
\frac{\sqrt{\pi}}{\gamma} \operatorname{erf}\left(\frac{1}{\gamma}\right) e^{1 / \gamma^{2}}=\varepsilon
$$

This equation determines a unique value for $1 / \gamma$ or equivalently $E_{0}$ as a function of $\varepsilon$. As $\varepsilon \rightarrow 0$ or $\varepsilon \rightarrow \infty$, the expression for $E_{0}$ can be evaluated asymptotically. We find that

$$
E_{0}=2-\frac{2 \varepsilon}{3}+O\left(\varepsilon^{2}\right) \quad \text { as } \varepsilon \rightarrow 0
$$

and

$$
E_{0} \sim \frac{4(\ln \varepsilon)}{\varepsilon} \text { as } \varepsilon \rightarrow \infty
$$

(See Fig. 1.) In summary, we have found

$$
t=\frac{L^{2}}{E_{0}}+O\left(L^{2-1 / n}\right)
$$

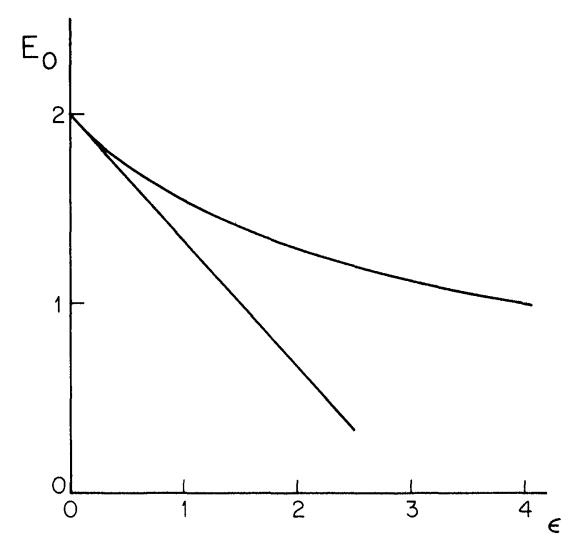

FIG. 1. The function $E_{0}(\varepsilon)$ is computed numerically using (2.35) and (2.36). As $\varepsilon \rightarrow 0$, the function approaches (2.37) (straight line in the figure). 
and

$$
u(x, L)=1-\frac{\operatorname{erf}(x / \gamma L)}{\operatorname{erf}(1 / \gamma)}+O\left(L^{-1 / n}\right) \text { for } 0<x<L
$$

where $\gamma$ is defined by (2.35) and $E_{0}(\varepsilon)$ satisfies (2.36). Figure 2 illustrates the results of $\S \S 2.1$ and 2.2 for the short time and long time behavior of the penetrant front. We see that there is a transition from the initial $t$ behavior (Case II) to the long time $t^{1 / 2}$ behavior (Fickian). For pharmaceutical applications it is desirable to maintain Case II behavior (also called zero-order release) as long as possible. Thus, we would like to understand the transition from Case II behavior. This is done in the next section for small $\varepsilon$.

2.3. Complete time history of the penetrant front for small $\varepsilon$. In this section, we describe the complete time evolution of the penetrant front by an asymptotic analysis of (2.15)-(2.19) valid for small $\varepsilon$ but for all times. Specifically, we seek a solution of these equations in the form

$$
\begin{gathered}
u(y, t, \varepsilon)=u_{0}(y, t)+\varepsilon u_{1}(y, t) \cdots, \\
L(t, \varepsilon)=L_{0}(t)+\varepsilon L_{1}(t) \cdots .
\end{gathered}
$$

Upon substituting (2.41) and (2.42) into (2.15)-(2.19) and equating like powers of $\varepsilon$, we obtain the following problem for $L_{0}$ and $u_{0}$ :

$$
\begin{gathered}
u_{0 y y}=0, \quad 0<y<1, \\
u_{0}=1 \quad \text { at } y=0, \\
L_{0} L_{0}^{\prime}=-u_{0 y} \quad \text { at } y=1, \\
L_{0}^{\prime}=u_{0}^{n} \quad \text { at } y=1, \\
L_{0}(0)=0 .
\end{gathered}
$$

The solution of (2.43) and (2.44) is given by

$$
u_{0}(y, t)=-A(t) y+1
$$

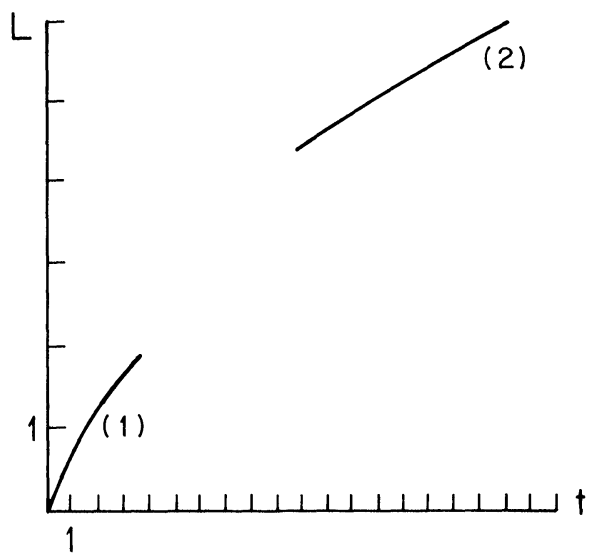

FIG. 2. Short time and long time behavior of the penetrant front. The short time behavior is described by equation (2.26) ( $n=1$ and $\left.\varepsilon=10^{-2}\right)$ and is represented by curve (1). The long time behavior is described by equation (2.39) where $E_{0}(\varepsilon)$ is given by (2.37) for small $\varepsilon\left(\varepsilon=10^{-2}\right)$. It is represented by curve (2). 
where $A(t)$ is an arbitrary function of $t$ to be determined using the conditions (2.45)-(2.47). From (2.45) and (2.46) we find

$$
\begin{gathered}
L_{0} L_{0}^{\prime}=A, \\
L_{0}^{\prime}=(1-A)^{n} .
\end{gathered}
$$

Eliminating $L_{0}^{\prime}$, we obtain the solution for $L_{0}=L_{0}(A(t))$

$$
L_{0}(A(t))=\frac{A}{(1-A)^{n}}
$$

where $0 \leqq A \leqq 1$ since $0 \leqq L_{0}<\infty$. Then using (2.50) and (2.51), we obtain a differential equation for $A(t)$. We integrate this equation using $A(0)=0$ (since $L(0)=0$ ) and find $t=t(A)$ :

$$
t(A)=\left[1+2(n-1) A-(1-A)^{2 n}\right] /\left[2(2 n-1)(1-A)^{2 n}\right] .
$$

Equations (2.51) and (2.52) give $L_{0}=L_{0}(t)$ in terms of the parameter $A$. For small times $(A \rightarrow 0)$ or large times $(A \rightarrow 1)$, we find the following asymptotic behaviors

$$
\begin{aligned}
& u(x, t, \varepsilon)=1-x+O(t x, \varepsilon), \\
& L(t, \varepsilon)=t-\frac{n}{2} t^{2}+O\left(t^{3}, \varepsilon\right)
\end{aligned}
$$

as $t \rightarrow 0$ and

$$
\begin{aligned}
& u(x, L, \varepsilon)=1-\frac{x}{L}+O\left(x L^{-1 / n-1}, \varepsilon\right), \\
& t=\frac{L^{2}}{2}+O\left(L^{2-1 / n}, \varepsilon\right)
\end{aligned}
$$

as $t \rightarrow \infty$. Note that the expressions given by (2.53) and (2.54) agree as $\varepsilon \rightarrow 0$ with the short times and large times solutions given by (2.25), (2.26) and (2.39), (2.40), respectively. (In (2.40), erf $(x / \gamma L) / \operatorname{erf}(1 / \gamma) \sim x / L$ because $\gamma \rightarrow \infty$ as $\varepsilon \rightarrow 0)$. It also confirms the use of $\tau=L^{-1 / n}$ as the small parameter for the expansion of $u(x, L)$ and $t(L)$.

As an illustration of the small $\varepsilon$ solution, we describe the case $n=1$. From (2.51) and (2.52), we find

$$
L_{0}(A)=\frac{A}{1-A} \quad \text { and } \quad t(A)=\frac{1}{2}\left[(1-A)^{-2}-1\right]
$$

or equivalently if we eliminate $A$,

$$
L_{0}(t)=-1+(1+2 t)^{1 / 2} \quad \text { and } \quad A(t)=\frac{L_{0}(t)}{L_{0}(t)+1} .
$$

Thus, using (2.48) we conclude that

$$
\begin{gathered}
u(x, t, \varepsilon)=-\frac{x}{(1+2 t)^{1 / 2}}+1+O(\varepsilon), \\
L(t, \varepsilon)=-1+(1+2 t)^{1 / 2}+O(\varepsilon) .
\end{gathered}
$$

Figures 3 and 4 represent $L(t)$ and $u(x, t)$, respectively.

We now consider the effect of changing $n$. Figure 5 represents $L(t)$ as a function of $t$ for $n=10^{-2}, 1$ and $10^{2}$, respectively. As $n \rightarrow 0$, we note that $L \sim t$ for longer periods 

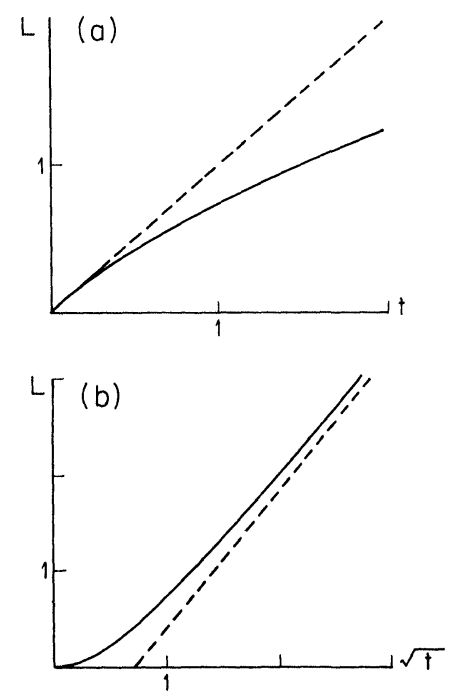

FIG. 3. Time history of the penetrant front as $\varepsilon \rightarrow 0$. In Figs. 3(a) and 3(b), $L(t)$ is represented as a function of $t$ and $\sqrt{t}$, respectively. $L(t)$ is given by equation (2.58). As $t \rightarrow 0, L(t)$ approaches the straight line $L(t)=t$ (broken line in Fig. 3(a)). As $t \rightarrow \infty, L(t)$ approaches the straight line $L(\sqrt{t})=\sqrt{2} \sqrt{t}-1$ (broken line in Fig. 3(b)).
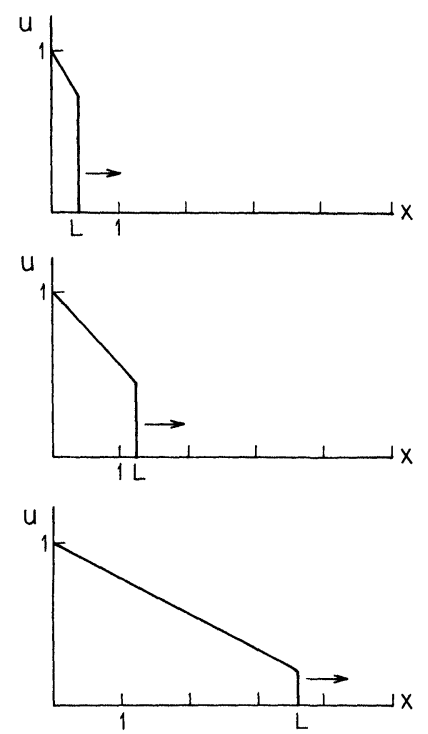

FIG. 4. Time history of the concentration as $\varepsilon \rightarrow 0 . u(x, t)$ is given by equation (2.57) and is represented as a function of $x$ for $t=0.5,2$ and 10 , respectively. For small $t, u \sim 1-x(0<x<t)$ and is quasi-stationary. For large $t, u \sim 1-x / \sqrt{2 t}(0<x<\sqrt{2 t})$ and has a Fickian behavior. 


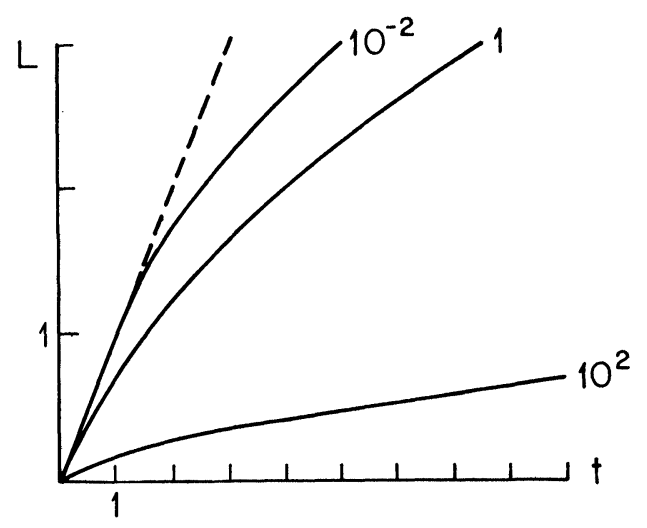

FIG. 5. Effect of $n, L(t)$ is obtained from (2.51) and (2.52) by varying A gradually from zero to one. The broken line corresponds to $L=t$, i.e., the short time behavior. The three curves correspond to the cases $n=10^{-2}$, 1 and $10^{2}$, respectively.

of time. This is in agreement with the short times result (2.53) since the coefficient of $t^{2}$ is proportional to $n$. If $n=0$, we directly obtain from (2.51) and (2.52) that $L=t$ for all times.

3. Sorption of a finite amount of swelling solvent. In this section, we study the time history of the solvent front when the polymer is exposed initially to a finite amount of penetrant. A constant concentration $C=C_{0}$ is no longer maintained at $X=0$ and the solvent-polymer system may approach an equilibrium at $C=C^{*}$. Because of this equilibrium, the time history of the interface is expected to be more complex. Instead of (2.1)-(2.5), the diffusion problem is now formulated by the following equations:

$$
\begin{gathered}
C_{T}=D C_{X X}, \quad 0<X<S(T), \\
C_{X}=0 \quad \text { at } X=0, \\
(C+K) S^{\prime}=-D C_{X} \quad \text { at } X=S(T), \\
S^{\prime}=k_{1}\left(C-C^{*}\right)^{n} \text { at } X=S(T), \\
S(0)=S_{i} \neq 0, \\
C(X, 0)=C_{i} .
\end{gathered}
$$

The evolution equations are the same as in $\S 2$. Equation (3.1) with (3.2) is Fick's diffusion equation subject to a zero flux boundary condition. Equation (3.3) is a mass balance equation at the front and was previously derived in $\S 2$. Equation (3.4) describes the interface kinetics. Problem (3.1)-(3.6) mainly deviates from our previous study by the boundary condition (3.2) (instead of $C=C_{0}$ at $X=0$ ) and the initial conditions (3.5) and (3.6) (instead of $S(0)=0$ ). In (3.6) we have assumed for simplicity that the initial concentration is constant. Note that the new problem depends on two control parameters: $C_{i}$ and $S_{i}$.

System (3.1)-(3.6) arises in modeling the formation and storage of a simple swelling controlled drug release system without volume change [7]. We have a slab of polymer with impermeable faces into which, at time zero, a finite and fixed amount of penetrant is injected to a depth $S_{i}$. For use, the impermeable-containing membrane is either removed or becomes permeable when placed in a dissolving solution. Of particular scientific interest is the time history of the diffusing front under the relevant driving kinetics. 
Equations (3.1)-(3.6) admit a useful conservation relation which we now derive. Upon integrating (3.1), we obtain

$$
\int_{0}^{S(T)} C_{T} d X=\int_{0}^{S(T)} D C_{X X} d X=D C_{X \mid X=S(T)}-D C_{X \mid X=0} .
$$

Then using (3.2) and (3.3), (3.7) can be written as

$$
\frac{d}{d T} \int_{0}^{S(T)} C d X+K \frac{d S}{d T}=0
$$

and thus

$$
\int_{0}^{S(T)} C d X+K S=Q
$$

where the constant $Q$ measures the quantity of the penetrant per unit length initially injected. Evaluating (3.9) at $T=0$, we find

$$
Q=\left(C_{i}+K\right) S_{i}
$$

Note, from (3.1)-(3.6) and then from (3.9), the existence of the equilibrium solution

$$
C(X, T)=C^{*}, \quad S(T)=\frac{Q}{C^{*}+K}=S_{\infty} .
$$

Thus, the finite amount of penetrant is used up, and the front comes to rest at the distance $S_{\infty}$ given by (3.11). We are interested in studying the time history of how $S(T)$ reaches $S_{\infty}$. To do this it will be convenient to introduce the following dimensionless variables:

$$
t=\frac{T}{\alpha}, \quad x=\frac{X}{\beta}, \quad u(x, t)=\frac{C(X, T)-C^{*}}{C_{i}-C^{*}}, \quad L(t)=\frac{S(T)}{\beta}
$$

where

$$
\alpha=\frac{Q}{k_{1}}\left(C_{i}-C^{*}\right)^{-n-1}, \quad \beta=Q\left(C_{i}-C^{*}\right)^{-1} .
$$

Then, the problem (3.1), (3.2), (3.4)-(3.6), and (3.9) becomes

$$
\begin{gathered}
u_{t}=\delta u_{x x}, \quad 0<x<L(t), \\
u_{x}=0 \quad \text { at } x=0, \\
L^{\prime}=u^{n} \quad \text { at } x=L(t), \\
\int_{0}^{L} u d x+\varepsilon^{-1} L=1, \\
L(0)=L_{i}=\frac{\varepsilon}{1+\varepsilon}, \\
u(x, 0)=1, \quad 0<x<L_{i},
\end{gathered}
$$

where

$$
\varepsilon=\frac{C_{i}-C^{*}}{C^{*}+K}, \quad \delta=\frac{D}{k_{1} Q}\left(C_{i}-C^{*}\right)^{1-n}
$$


and $L_{i}=S_{i} / \beta$ has been evaluated using (3.10) and the definition of $\varepsilon$. In these variables the equilibrium solution (3.11) becomes

$$
u(x, t)=0, \quad L(t)=\varepsilon .
$$

The solution of (3.13)-(3.18) depends on the values of two key parameters, $\varepsilon$ and $\delta$. $\varepsilon$ is defined as in $\S 2$ and is proportional to the deviation $C_{i}-C^{*}$. It can thus be controlled by changing the initial concentration $C_{i} . \delta$ is a new control parameter and is inversely proportional to $Q$, the initial quantity of penetrant. Since $Q$ is proportional to $S_{i}, \delta$ can be changed by changing $S_{i}$ without modifying $\varepsilon$. Of particular physical interest is the limit $S_{i} \rightarrow 0$. This limit can be achieved experimentally in two ways depending on the value of $C_{i}$. First, we may keep $C_{i}=O(1)$ fixed and consider the limit $S_{i} \rightarrow 0$. This is equivalent to requiring $\delta=O\left(S_{i}^{-1}\right) \rightarrow \infty, \varepsilon$ fixed. Another possibility is to consider the simultaneous limit $S_{i} \rightarrow 0$ and $C_{i} \rightarrow \infty$ which leads to the condition $\varepsilon \rightarrow \infty$ and $\delta=O(1)$ (if $C_{i}=O\left(S_{i}^{-1 / n}\right)$ ). These cases are of practical interest and we shall analyze them in detail. As in $\S 2$, we first introduce (2.14) and reformulate (3.13)-(3.18):

$$
\begin{gathered}
L^{2} u_{t}=\delta u_{y y}+y L L^{\prime} u_{y}, \quad 0<y<1, \\
u_{y}=0 \quad \text { at } y=0, \\
L^{\prime}=u^{n} \quad \text { at } y=1, \\
\left(\int_{0}^{1} u d y+\varepsilon^{-1}\right) L=1, \\
L(0)=L_{i}(\varepsilon), \\
u(y, 0)=1, \quad 0<y<1 .
\end{gathered}
$$

To investigate the limit $\delta \rightarrow \infty$, we seek a solution of these equations in the form

$$
\begin{gathered}
u(y, t, \delta)=u_{0}(y, t)+\delta^{-1} u_{1}(y, t)+\cdots, \\
L(t, \delta)=L_{0}(t)+\delta^{-1} L_{1}(t)+\cdots .
\end{gathered}
$$

Inserting (3.27) and (3.28) into (3.21)-(3.26) and equating to zero the coefficients of each power of $\delta$, we find the following problem for $u_{0}$ and $L_{0}$ :

$$
\begin{gathered}
u_{0 y y}=0, \quad 0<y<1, \\
u_{0 y}=0, \quad y=0, \\
L_{0}^{\prime}=u_{0}^{n}, \quad y=1, \\
\left(\int_{0}^{1} u_{0} d y+\varepsilon^{-1}\right) L_{0}=1, \\
L_{0}(0)=L_{i}, \\
u(y, 0)=1, \quad 0<y<1 .
\end{gathered}
$$

We first determine the solution of (3.29) and (3.30)

$$
u_{0}(y, t)=B(t)
$$

where $B$ is an arbitrary function of $t$. Introducing (3.36) into (3.31) and (3.32), we have

$$
\begin{gathered}
L_{0}^{\prime}=B^{n}, \\
\left(B+\varepsilon^{-1}\right) L_{0}=1 .
\end{gathered}
$$


From (3.38) we find $B\left(L_{0}\right)$

$$
B\left(L_{0}\right)=\frac{1-\varepsilon^{-1} L_{0}}{L_{0}} .
$$

Then, using (3.37), we obtain $L_{0}(t)$ implicitly

$$
t=\int_{L_{i}}^{L_{0}} \frac{l^{n} d l}{\left(1-\varepsilon^{-1} l\right)^{n}} .
$$

From $u(y, 0) \sim B\left(L_{0}(0)\right)$ and the expression of $L_{i}(\varepsilon)$ given in (3.17), we have verified that the initial condition (3.35) is satisfied. To discuss the interest of our result, we consider the case $n=1$. From (3.40) we then obtain

$$
t=-\varepsilon\left(L_{0}-L_{i}\right)-\varepsilon^{2} \ln \left(\frac{\varepsilon-L_{0}}{\varepsilon-L_{i}}\right) .
$$

Since $L(t, \delta)=L_{0}(t)+O\left(\delta^{-1}\right)$ for all $t$, we may describe, using (3.41), the short time and long time behavior

$$
\begin{gathered}
L-L_{i} \sim t \text { for small } t \\
L-\varepsilon \sim-\frac{\varepsilon^{2}}{1+\varepsilon} e^{-t / \varepsilon^{2}} e^{-\varepsilon /(1+\varepsilon)} \text { for large } t .
\end{gathered}
$$

Figure 6 represents $L(t)$ as a function of $t$ for two different values of $\varepsilon$.

To complete our analysis we have studied the limits $\varepsilon \rightarrow \infty$ and $\varepsilon \rightarrow 0$ but $\delta$ fixed. These two limits are briefly described in Appendix A and B, respectively. It is interesting to note that the limit $\varepsilon \rightarrow \infty, \delta$ fixed leads to the equations analyzed by Cohen and Goodhart [5]. This is not surprising since the limit $\varepsilon \rightarrow \infty, \delta$ fixed means $C_{i} \rightarrow \infty$, $S_{i}=O\left(C_{i}^{-n}\right) \rightarrow 0$ and the problem analyzed by Cohen and Goodhart concentrated on the case $S_{i}=0$ and $C(X, 0)=C_{i} \delta(X)$ where $\delta(x)$ is the delta function.

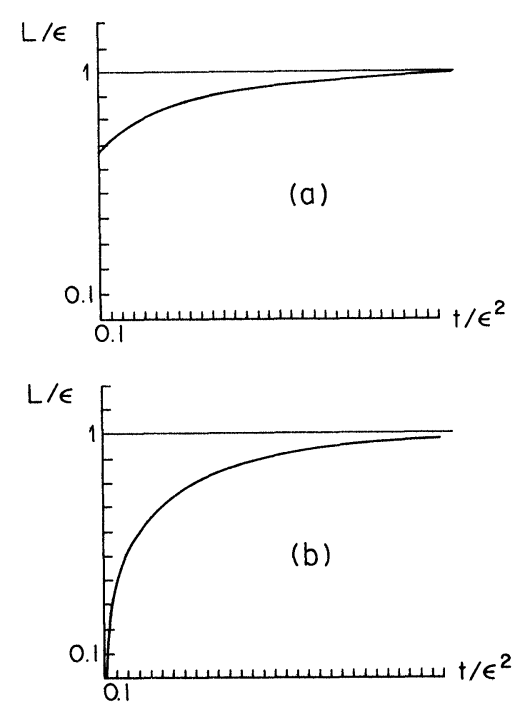

FIG. 6. Time history of the penetrant front as $\delta \rightarrow \infty$. Figs. $6(\mathrm{a})$ and $6(\mathrm{~b})$ represent $L(t) / \varepsilon$ as a function $t / \varepsilon^{2}$ for $\varepsilon=0.5$ and 10 , respectively. 
4. Discussion. In this paper, we have formulated and studied two different free boundary problems describing the transport of a solvent through a glassy polymer. We have analyzed the time history of the moving front by exploring different asymptotic limits (short time, long time and the steady state approximation [2, p. 310]). We expect that our analysis will be useful in studying other free boundary problems characterized by changing time histories (for example, the vertical infiltration of water into an initially dry soil [10]). Recently, other mathematical or numerical approaches have been proposed to analyze (2.1)-(2.5) [11], [12].

For many applications of controlled release, it is desirable that the release rate be constant. To achieve this objective, we would like to observe the short time behavior $S(T) \sim T$ during a long period of time. To this end, it is interesting to analyze the short time behavior in detail. In the first case (constant reservoir of solvent), we find from (2.26) and $n=1$ that $t=t_{M}=2 /(1+\varepsilon)$ represents the maximum (dimensionless) time interval for zero order release. If $t \ll t_{M}, L(t)=t+O\left(t^{2}\right)$ but if $t>O\left(t_{M}\right)$, the approximation (2.26) is no longer valid. Using the definitions (2.7) with $n=1$, we find that the maximum time interval is given by

$$
T_{M}=\frac{D}{\bar{C}^{2} k_{1}^{2}} \frac{2}{\varepsilon(1+\varepsilon)}
$$

which is large when $\epsilon \ll 1$. In the second case (finite amount of solvent), we find from (3.41) that the short time behavior is given by

$$
L-L_{i}=t-\frac{t^{2}}{2 L_{i}^{2}}+O\left(t^{3}\right)
$$

(as $\delta \rightarrow \infty$ and $n=1$ ) where $L_{i}(\varepsilon)$ is defined by (3.17). From (4.2), we then obtain that the maximum (dimensionless) time interval for zero order release is $t_{m}=2 L_{i}^{2}$. Only if $t \ll t_{M}, L-L_{i}=t+O\left(t^{2}\right)$. Using the definitions (3.12), (3.19) with $n=1$ and (2.7d), we find that the maximum time interval is

$$
T_{M}=\frac{D}{k_{1}^{2} \bar{C}^{2}} \frac{1}{\delta} \frac{2}{(1+\varepsilon)^{2}} .
$$

We conclude that $T_{m}$ is an $O(1)$ quantity if $\varepsilon$ is sufficiently small.

Appendix A. The limit $\varepsilon \rightarrow \mathbf{0}, \boldsymbol{\delta}$ fixed. We consider (3.13)-(3.15), (3.17), and (3.18) with $n=1$. Instead of the integral condition (3.16), we shall use the mass balance equation (3.3) which is more convenient for this case. In terms of the dimensionless variables, this condition is given by

$$
\left(\varepsilon^{-1}+u\right) L^{\prime}=-\delta u_{x} \quad \text { at } x=L(t) .
$$

We now analyze the limit $\varepsilon \rightarrow 0$ of these equations by introducing the following expansions of $u(x, t, \varepsilon)$ and $L(t, \varepsilon)$ :

$$
\begin{aligned}
& u(x, t, \varepsilon)=u(\bar{x}, \bar{t}, \varepsilon)=u_{0}(\bar{x}, \bar{t})+\varepsilon u_{1}(\bar{x}, \bar{t})+\cdots, \\
& L(t, \varepsilon)=L(\bar{t}, \varepsilon)=L_{i}(\varepsilon)+\varepsilon^{2} L_{2}(\bar{t})+\varepsilon^{3} L_{3}(\bar{t})+\cdots
\end{aligned}
$$

where $\bar{x}$ and $\bar{t}$ are defined by

$$
\begin{aligned}
& \bar{x}=\frac{x}{\varepsilon}, \\
& \bar{t}=\frac{t}{\varepsilon^{2}} .
\end{aligned}
$$


The expansion of $L(t, \varepsilon)$ is suggested by the fact that $L(\infty)-L_{i}(\varepsilon)=\varepsilon-\varepsilon /(1+\varepsilon)=$ $O\left(\varepsilon^{2}\right)$. Equation (3.15) then requires that $L$ is a function of $t / \varepsilon^{2}$ and suggests (A5). Since $L$ is an $O(\varepsilon)$ quantity, we introduce the scaling (A4). The leading order problem for $u_{0}(\bar{x}, \bar{t})$ and $L_{2}(\bar{t})$ is given by

$$
\begin{gathered}
u_{0 \bar{t}}=\delta u_{0 \bar{x} \bar{x}}, \quad 0<\bar{x}<1, \\
u_{0 \bar{x}}=0 \quad \text { at } \bar{x}=0, \\
L_{2}^{\prime}=u_{0} \quad \text { at } \bar{x}=1, \\
L_{2}^{\prime}=-\delta u_{0 \bar{x}} \quad \text { at } \bar{x}=1, \\
u_{0}(\bar{x}, 0)=1, \quad 0<\bar{x}<1, \\
L_{2}(0)=0
\end{gathered}
$$

where $\delta$ is $O(1)$ and has been evaluated at $\varepsilon=0$ with $Q=\left(C^{*}+K\right) S_{i}$. The solution is easily found and is given by

$$
\begin{aligned}
& u_{0}(\bar{x}, \bar{t})=\sum_{n: 1}^{\infty} c_{n} e^{-k_{n}^{2} \delta \bar{t}} \cos \left(k_{n} \bar{x}\right), \\
& L_{2}(\bar{t})=\sum_{n: 1}^{\infty} c_{n} \frac{\cos \left(k_{n}\right)}{\delta k_{n}^{2}}\left(1-e^{-k_{n}^{2} \delta \bar{t}}\right)
\end{aligned}
$$

where $k_{n}$ satisfies the transcendental equation

$$
\cot \left(k_{n}\right)=\delta k_{n}
$$

and $c_{n}$ is obtained using the initial conditions

$$
c_{n}=\frac{2}{k_{n}} \frac{\sin \left(k_{n}\right)}{\left(1+\sin \left(2 k_{n}\right) / 2 k_{n}\right)} .
$$

As $\delta \rightarrow \infty, k_{1} \sim \delta^{-1 / 2}, k_{n} \sim n \pi(n \geqq 2), c_{1} \sim 1$ and $c_{n}=O\left(\delta^{-1}\right)(n \geqq 2)$. Consequently,

$$
L_{2}(\bar{t}) \sim 1-e^{-\bar{t}}
$$

and

$$
u_{0}(\bar{x}, \bar{t}) \sim e^{-\bar{t}}
$$

which match the results of our previous analysis when $\delta \rightarrow \infty$ and then $\varepsilon \rightarrow 0$.

Appendix B. The limit $\epsilon \rightarrow \infty \delta$ fixed. We consider (3.13)-(3.18) with $n=1$. We want the two terms at the left-hand side of (3.16) to remain $O(1)$ as $\varepsilon \rightarrow \infty$. This implies that $L=O(\varepsilon)$ (and thus $x=O(\varepsilon)$ ) and $u=O\left(\varepsilon^{-1}\right)$. Then from (3.15), we must require $t=O\left(\varepsilon^{2}\right)$. This suggests the following expansion of $u(x, t, \varepsilon)$ and $L(t, \varepsilon)$ :

$$
\begin{gathered}
u(x, t, \varepsilon)=u(\bar{x}, \bar{t}, \varepsilon)=\varepsilon^{-1}\left(u_{0}(\bar{x}, \bar{t})+\varepsilon u_{1}(\bar{x}, \bar{t}) \cdots\right) \\
L(t, \varepsilon)=L(\bar{t}, \varepsilon)=\varepsilon\left(L_{0}(\bar{t})+\varepsilon L_{1}(\bar{t}) \cdots\right)
\end{gathered}
$$

where $\bar{t}$ and $\bar{x}$ are defined by

$$
\bar{t}=\frac{t}{\varepsilon^{2}}, \quad \bar{x}=\frac{x}{\varepsilon} .
$$


Introducing (B1)-(B3) into (3.13)-(3.18), we obtain the following problem for $u_{0}(\bar{x}, \bar{t})$ and $L_{0}(\bar{t})$ :

$$
\begin{gathered}
u_{0 \bar{t}}=\delta u_{0 \bar{x} \bar{x}}, \quad 0<\bar{x}<L_{0}(\bar{t}), \\
u_{0 \bar{x}}=0 \quad \text { at } \bar{x}=0, \\
L_{0}^{\prime}=u_{0} \quad \text { at } x=L_{0}(\bar{t}), \\
\int_{0}^{L_{0}} u_{0} d \bar{x}+L_{0}=1, \\
L_{0}(0)=0 \\
u_{0}(\bar{x}, 0)=\delta(\bar{x})
\end{gathered}
$$

where $\delta(\bar{x})$ is the Dirac delta function. These equations have been analyzed by Cohen and Goodhart [5].

Acknowledgment. D. S. Cohen thanks the members of the Center for Nonlinear Studies at Los Alamos for sponsoring and encouraging his interactions with various groups at Los Alamos.

\section{REFERENCES}

[1] T. Alfrey, E. F. Gurnee, And W. G. Lloyd, Diffusion in glassy polymers, J. Polymer Sci. Part C, 12 (1966), pp. 249-261.

[2] J. Crank, The Mathematics of Diffusion, 2nd ed., Oxford University Press, London, Oxford, 1976.

[3] D. S. CoHen, Theoretical models for diffusion in polymers. II, J. Polymer Sci., Polymer Phys. Edn., 22 (1984), pp. 1001-1009.

[4] H. L. FrisCH, Sorption and transport in glassy polymers, Polymer Engrg. and Sci., 20 (1980), pp. 2-13.

[5] D. S. COHEN AND C. GoOdHART, Sorption of a finite amount of swelling solvent in a glassy polymer, J. Polymer Sci., Polymer Phys. Edn., 25 (1987), pp. 611-617.

[6] G. Astarita AND S. Joshi, Sample dimension effects in the sorption of solvents in polymers-a mathematical model, J. Membrane Sci., 4 (1978), pp. 165-182.

[7] R. W. Korsmeyer AND N. A. Peppas, Macromolecular and modeling aspects of swelling-controlled systems, in Controlled Release Delivery Systems, T. J. Roseman and S. Z. Mansdorf, eds., Marcel Dekker, New York, 1983.

[8] G. Astarita AND G. C. SARti, A class of mathematical models for sorption of swelling solvents in glassy polymers, Polymer Engrg. and Sci., 18 (1978), pp. 388-395.

[9] R. LANGer, Polymeric delivery systems for controlled drug release, Chem. Engrg. Commun., 6 (1980), pp. $1-48$.

[10] D. Hillel, Introduction to Soil Physics, Academic Press, New York, 1982, p. 219.

[11] A. Fasano, G. H. Meyer, And M. Primicerio, On a problem in the polymer industry: theoretical and numerical investigation of swelling. SIAM J. Math. Anal., 17 (1986), pp. 945-960.

[12] D. ANDREUCCI AND R. RiCCl, A free boundary problem arising from sorption of solvents in glassy polymers, Quart. Appl. Math., XLIV (1987), pp. 649-657. 